\title{
Numerical simulation of the influence of the orifice aperture on the flow around a teeth-shaped obstacle.
}

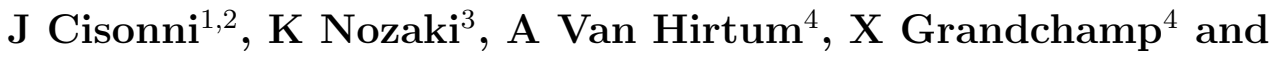 \\ $\mathrm{S} \mathrm{Wada}^{3}$ \\ ${ }^{1}$ The Center for Advanced Medical Engineering and Informatics, Osaka University, \\ 1-3 Machikaneyama, Toyonaka, Osaka, 560-8531, JAPAN \\ ${ }^{2}$ Fluid Dynamics Research Group, Department of Mechanical Engineering, Curtin \\ University, GPO Box U1987, Perth WA 6845, AUSTRALIA \\ ${ }^{3}$ Graduate School of Engineering Science, Osaka University, 1-3 Machikaneyama, \\ Toyonaka, Osaka, 560-8531, JAPAN \\ ${ }^{4}$ GIPSA-lab, UMR CNRS 5216, Grenoble University, 11 rue des Mathematiques , BP \\ 46, 38402 Grenoble Cedex, FRANCE \\ E-mail: julien.cisonni@gmx.com
}

\begin{abstract}
The sound generated during the production of the sibilant [s] results from the impact of a turbulent jet on the incisors. Several geometric characteristics of the oral tract can affect the properties of the flow-induced noise so that the characterization of the influence of different geometric parameters on the acoustic sources properties allows determining control factors of the noise production. In this study, a simplified vocal tract/teeth geometric model is used to numerically investigate the flow around a teeth-shaped obstacle placed in a channel and to analyze the influence of the aperture at the teeth on the spectral properties of the fluctuating pressure force exerted on the surface of the obstacle, which is at the origin of the dipole sound source. The results obtained for $\mathrm{Re}=4000$ suggest that the aperture of the constriction formed by the teeth modifies the characteristics of the turbulent jet downstream of the teeth. Thus, the variations of the flow due to the modification of the constriction aperture lead to variations of the spectral properties of the sound source even if the levels predicted are lower than during the production of real sibilant fricative.
\end{abstract}

Keywords: Sibilant [s], Physical modeling, Flow-induced noise, Turbulence 


\section{Introduction}

The sibilant $[\mathrm{s}]$ is an unvoiced sound of speech which belongs to the fricative category. This type of sound results from the impact of a turbulent jet, formed in the vocal tract, on the incisors. The aeroacoustic phenomena involved in the production of this sound are complex. However, experimental studies allowed the characterization of the main physical mechanisms and in particular the strong acoustic source resulting from the presence of the teeth in a turbulent flow (Shadle 1985). The comparison made by Badin (1989) between spectra of fricatives obtained experimentally and by synthesis based on simplified area functions showed that the computed transfer functions were in good agreement with the measured data for the sibilant [s]. Fletcher and Newman (1991) investigated the influence of the location of the sibilant groove on the sound produced. Despite the variability in the place of articulation, the resulting sounds can be perceived as a [s] by listeners. Nissen and Fox (2005) carried out an analysis of spectral parameters of fricatives produced by adults and developing children and showed an increase in the acoustic distinction between $[\mathrm{s}]$ and the other sounds as the age of speaker increased. Moreover, Catford (1977) pointed out the increase in the high frequency spectral energy of sibilant produced by speakers wearing dentures.

The modeling of the turbulent flow within the cavities and the constrictions of the vocal tract is essential to characterize the acoustic sources of the fricatives, but remains very complex. Indeed, the shape of the vocal tract depends on several parameters which can have a strong influence on the flow properties and consequently on the sound produced. From vorticity predictions obtained with a Large-Eddy Simulation model and a simplified description of the vocal tract, Ramsay and Shadle (2006) suggest that aeroacoustic models of the turbulence in fricatives with a small number of geometric parameters can be developed, but also that using very simplified description of turbulent jet does not seem to be relevant for the modeling of fricatives. Moreover Krane (2005) strongly suggests that the interaction of the turbulent jet with the oral tract geometry is generating the noise from the pressure forces induced on the vocal tract walls. Thus, the discontinuities in the vocal tract shape appear to have a key role in the fricative sound production. Complete aeroacoustic models of the sibilant $[\mathrm{s}]$ production using simplified (Howe and McGowan 2005) or realistic 2-D (Anderson, Green and Fels 2009) and 3-D (Nozaki, Akiyama, Shimojo, Maeda and Tamagawa 2005, Nozaki 2005) geometries of the vocal tract and the teeth were proposed, but the influence of geometric parameters on the properties of the sound generated was not clearly determined.

Experimental in-vivo (Runte, Lawerino, Dirksen, Bollmann, Lamprecht-Dinnesen and Seifert 2001) and in-vitro (Shadle, Berezina, Proctor and Iskarous 2008) studies showed that small variations of the position of the tongue and the incisors can lead to significant changes in the spectral characteristics of the sound generated and therefore in the type of speech sound produced. The flow-induced sound generation also appears sensitive to the three-dimensional details of vocal tract geometry (Shadle 1991). Moreover, inverse approaches, as proposed by Sorokin (1994), to determine articulatory 
parameters from fricative spectral analysis, rely on an adequate modeling of the noise source. Thus, a systematic analysis of the influence of different geometric parameters on the properties of the sound generated, from numerical aeroacoustic simulation, appears to be a necessary phase in the development of a physical model of fricatives. Even if simplified geometrical models of the vocal tract remain unable to predict unvoiced sounds of speech (Shadle 1991), they are still useful to analyze the physics involved in the production of these sounds. The use of a simplified vocal tract/teeth obstacle allows focusing the study on one particular phenomenon which can be isolated. Moreover, it allows carrying out in-vitro experiments using the same geometry (Grandchamp, Van Hirtum, Nozaki, Cisonni, Pelorson and Shimojo 2010) which can be compared to results obtained from numerical simulations in order to provide an experimental validation of the physical modeling. Such kind of comparison was presented by Van Hirtum, Grandchamp, Pelorson, Nozaki and Shimojo (2010) for the near flow field around a teeth-shaped obstacle and conclude that even if experimental and simulated flow time-average features present the same tendencies, some discrepancies appear when a quantitative comparison of the properties is considered and that these discrepancies could be underlain by the limited modeling of the jet downstream of the obstacle.

In this paper, a simplified vocal tract/teeth geometric model is used to carry out a numerical study on the flow-induced noise source generated by a teeth-shaped obstacle placed in a channel. This teeth-shaped obstacle relevant to the incisors geometry was previously used by Van Hirtum et al. (2010) in order to simulate the turbulence generated by this obstacle. However, in this study, the geometric model includes a domain which represents the free field downstream of the obstacle and allows simulating the behavior of the turbulent jet resulting from the presence of the obstacle in the flow. This paper particularly investigates the influence of the aperture of the constriction formed by the obstacle on the resulting sound source. It aims at establishing a link between the aperture at the teeth and the properties of the noise source involved in the sibilant $[\mathrm{s}]$ production. Considering that the presence of the teeth as an obstacle in the flow is a strong acoustic source (Shadle 1985), that only the interactions of the flow with the surrounding walls produce sound (Krane 2005) and that the main acoustic source on ducts walls is of dipole type (Heller and Widnall 1970, Nelson and Morfey 1981), a frequency analysis of the fluctuating pressure force on the surface of the teeth-shaped obstacle is carried out for five different constriction apertures from numerical flow simulations. The geometrical model considered allows focusing the study on the dipole sound source on a generic teeth-shaped obstacle due to the separation of the flow and the formation of a jet downstream of this obstacle. This constitutes only one of the phenomena involved in the production of the sibilant $[\mathrm{s}]$ and other fricative sounds, and some constriction apertures considered are larger than the aperture between the teeth during the sibilant $[\mathrm{s}]$ production. Consequently, the spectral properties of the dipole sound source predicted differ from the one observed for the real sibilant $[\mathrm{s}]$ since they also depend on the acoustic resonances of the vocal tract and the sound radiation at the lips. 
Table 1. Constant geometric parameters of the model.

\begin{tabular}{lll}
\hline Parameter & Symbol & Value \\
\hline Channel Upstream Length & $L_{\text {up }}$ & $80 \mathrm{~mm}$ \\
Channel Downstream Length & $L_{\text {down }}$ & $11 \mathrm{~mm}$ \\
Constriction Length & $L_{T}$ & $1.25 \mathrm{~mm}$ \\
Channel Height & $H$ & $25 \mathrm{~mm}$ \\
Channel Width & $W$ & $101 \mathrm{~mm}$ \\
Obstacle Upstream Angle & $\alpha_{1}$ & $107^{\circ}$ \\
Obstacle Downstream Angle & $\alpha_{2}$ & $90^{\circ}$ \\
Downstream Domain Reference Length & $L_{\text {ext }}^{\text {ref }}$ & $330 \mathrm{~mm}$ \\
Downstream Domain Border Height & $H_{\text {ext }}$ & $30 \mathrm{~mm}$ \\
Downstream Domain Border Width & $W_{\text {ext }}$ & $10 \mathrm{~mm}$ \\
Downstream Domain Vertical Angle & $\beta_{\text {ext }}$ & $20^{\circ}$ \\
Downstream Domain Horizontal Angle & $\theta_{\text {ext }}$ & $10^{\circ}$ \\
\hline
\end{tabular}

\section{Numerical simulations}

Numerical flow simulations were carried out using the simplified vocal tract/teeth geometrical model described in Section 2.1. The Large-Eddy Simulation method for incompressible flow was used to simulate the turbulent flow within the constriction formed by the teeth-shaped obstacle. The conditions and the parameters used for this modeling are given in Section 2.2 and Section 2.3. The flow-induced noise sources were derived from the results of the flow simulations as described in Section 2.4.

\subsection{Geometry definition}

The geometric model used to carry out the numerical simulations is a simplification of the geometry of the oral cavity and the incisors. A generic teeth-shaped obstacle (Van Hirtum et al. 2010) is placed in a rectangular channel in order to create a constriction of which the height is $H_{C}$. In the following, the constriction height to the channel height ratio $H_{C} / H$ is the varying parameter considered. A cavity representing a free field is added downstream of the channel. A description of the geometric model is presented in figure 1 and figure 2 . The values of the geometric parameters considered are listed in table 1 and table 2. The shape of the obstacle is defined with sizes and angles derived from morphological data relevant to the upper incisors (Fredericks 1974, Ellis and McNamara 1986, Rudolph, Dominguez, Ahn and Thinh 1998, Magne, Gallucci and Belser 2003).

The other geometric parameters are set in order to design a model suitable for numerical turbulent flow simulation so that the values are chosen according to a priori known properties of the simulated flows and several preliminary simulations. The length of the channel upstream of the teeth-shaped obstacle is set in order to ensure that a parabolic velocity profile is fully developed upstream of the constriction, since a uniform velocity profile is set at the channel entrance (see Section 2.2). The value of the channel 


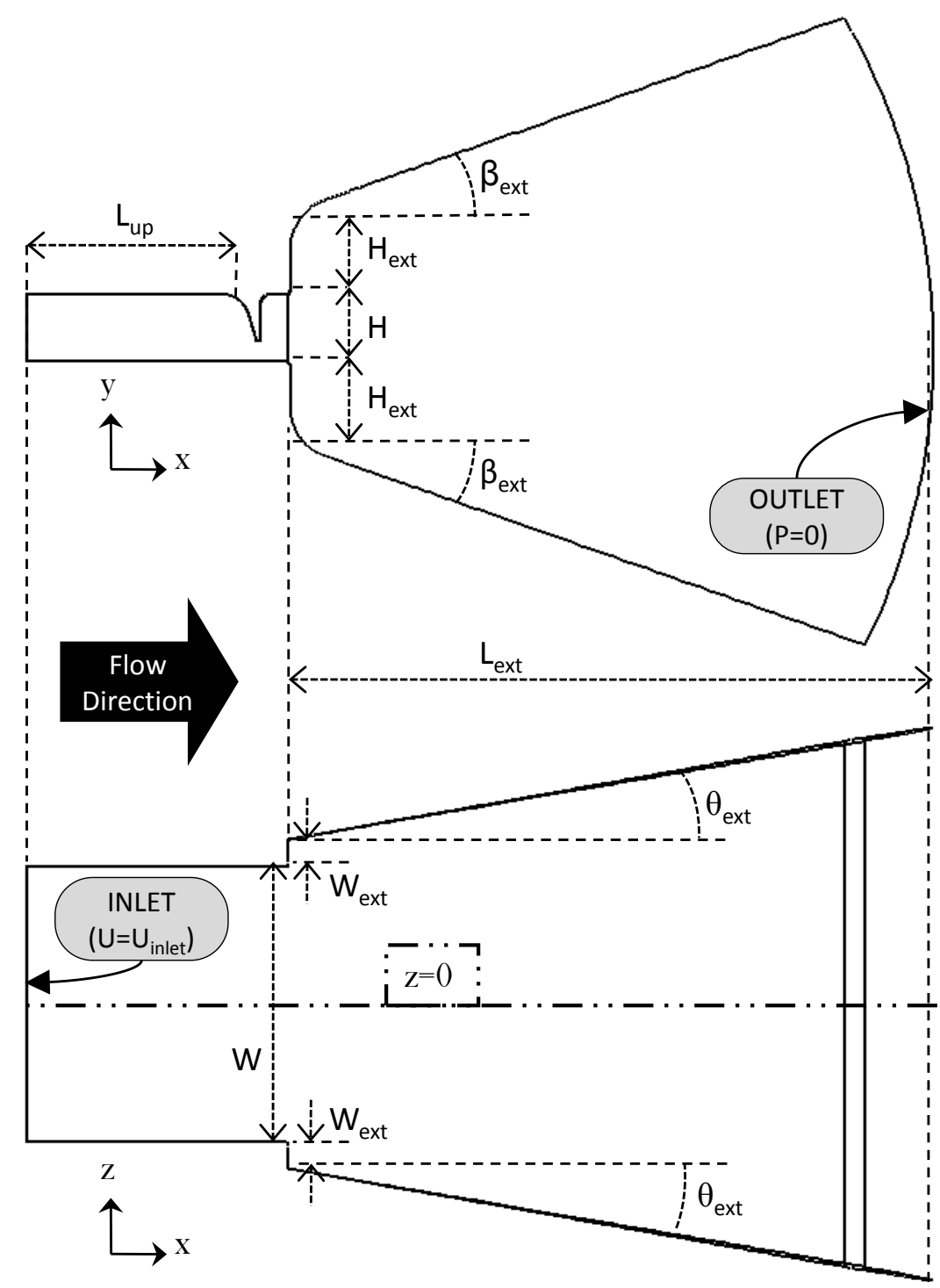

Figure 1. Description of the geometric model in $(x, y)$ and $(x, z)$ planes. The cutting plane $(x, y, z=0)$ is indicated with the dot-dashed line

width to height ratio $(W / H \approx 4)$ ensures that the simulated flow in the middle of the channel is weakly affected by the side walls and allows considering that the flow properties will mainly vary in the $(x, y)$ plane. The free field domain downstream of the channel allows the simulation of the flow behavior downstream of the constriction and in particular the formation of a turbulent jet due to the obstacle in the channel. The surrounding walls in the downstream domain are placed sufficiently far from the center of the channel so that the flow simulated in this domain can be considered as a free jet. Moreover, the effective length of the jet potential core is expected to decrease as the constriction aperture decreases. Thus, from preliminary flow simulations and in order to reduce the computational cost, the length of the downstream domain $L_{e x t}$ is thus adapted according to the constriction aperture $H_{C}$, so that: 


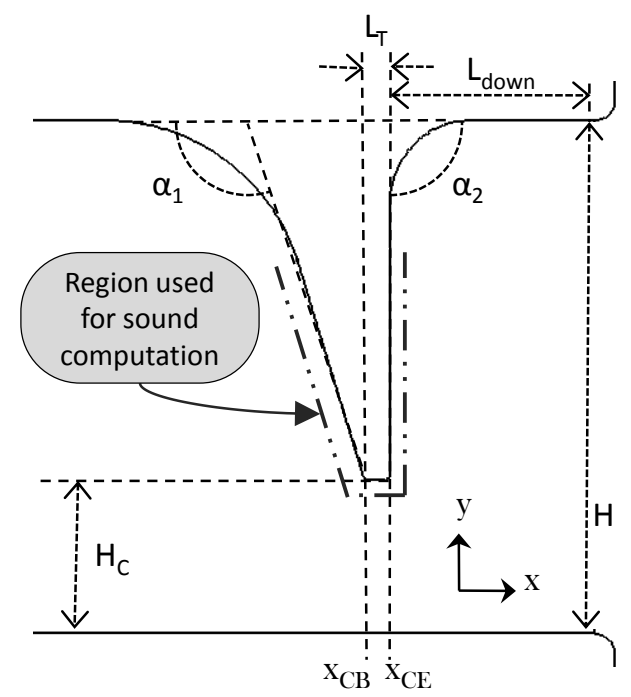

Figure 2. Description of the teeth-shaped obstacle in $(x, y)$ plane.

Table 2. Varying geometric parameters of the model.

\begin{tabular}{lll}
\hline Constriction Height & Constriction Height Ratio & $\begin{array}{l}\text { Downstream Domain Length } \\
H_{C}(\mathrm{~mm})\end{array}$ \\
$H_{C}(\mathrm{~mm})$ & 6 & 148 \\
1.5 & 8 & 170 \\
2.0 & 10 & 190 \\
2.5 & 20 & 270 \\
5.0 & 30 & 330 \\
7.5 & & \\
\hline
\end{tabular}

$$
L_{e x t}=L_{e x t}^{r e f} \sqrt{\frac{10}{3} \frac{H_{C}}{H}} .
$$

Finally, all the corners around the obstacle and at the channel exit are rounded to obtain a smooth geometry. This allows avoiding singular points which can affect the computation.

\subsection{Meshing and boundary conditions}

The spatial discretization of the whole flow domain was carried out using Gridgen v15 (Pointwise, Inc.). A structured mesh is defined using hexahedral 8-node finite elements. Smaller elements are used near the walls to take into account the boundary layer effects whereas the mesh is coarser in the body of the flow. Furthermore, the mesh is finer within the constricted region and in the region where the turbulent jet is a priori located. The lengths in $x$ and $y$ directions, respectively $\Delta_{x}$ and $\Delta_{y}$, of each element are set in order to ensure the stability of the flow model so that, for each element, the aspect ratio verifies:

$$
0.4<\frac{\Delta_{x}}{\Delta_{y}}<2.5
$$


Table 3. Meshing parameters for the numerical simulation.

\begin{tabular}{lll}
\hline Constriction Height Ratio & Total Number of Elements & $\begin{array}{l}\text { Minimum Elements Size } \\
H_{C} / H(\%)\end{array}$ \\
\hline 6 & $4,554,108$ & $\Delta_{x} / \Delta_{y}(\mathrm{~m})$ \\
8 & $4,561,656$ & $2.2 \times 10^{-5} / 9.5 \times 10^{-6}$ \\
10 & $4,661,919$ & $3.0 \times 10^{-5} / 1.2 \times 10^{-5}$ \\
20 & $4,851,656$ & $3.3 \times 10^{-5} / 1.4 \times 10^{-5}$ \\
30 & $5,420,868$ & $5.4 \times 10^{-5} / 2.2 \times 10^{-5}$ \\
\hline
\end{tabular}

Only low variations of the flow properties in the $z$-direction are expected so that the elements size in that direction is larger. The size of the filter used to filter the flow equations in the Large-Eddy Simulation method is derived automatically from the volume of the elements (see Section 2.3). Moreover, in this method, the spatial filtering leads to a low-pass frequency filtering (Sagaut 2005). Since the purpose of this study is to characterize the flow-induced sound, the volume of the elements is set in order to reduce the impact of the filtering in the frequency range considered $[0-25000 \mathrm{~Hz}]$.

The elements size is adapted according to the constriction aperture so that the narrower the aperture, the finer the mesh is in the constricted region. This leads to an increase in the number of elements in the channel when the constriction aperture is decreased. However, as explained in Section 2.1, the length of the downstream domain is reduced when the constriction aperture is decreased so that the number of elements in the downstream domain is decreased. Thus, the total number of elements is comprised between 4,500,000 and 5,500,000 in the five different geometries considered. Table 3 indicates the total number of elements as well as the minimum elements stream-wise and span-wise sizes, $\Delta_{x}$ and $\Delta_{y}$, corresponding to each constriction aperture. Several preliminary simulations carried out using different mesh densities showed that the number of elements is sufficient to consider the results obtained as grid-independent.

The boundary conditions specified in the model are a uniform velocity profile at the channel entrance (inlet) and a static pressure equal to 0 at the downstream domain exit (outlet) (see figure 1). To ensure the stability of the computation, an additional condition which set the normal velocity to zero when reverse flow is detected is added to prevent from backflow at the outlet. The inlet velocity is set to $2.4 \mathrm{~m} \cdot \mathrm{s}^{-1}$ to obtain a typical Reynolds number value of 4000 from the channel entrance height $H$. Except for the inlet and outlet surfaces, the no-slip wall condition is specified for all the boundaries of the channel and the downstream domain.

\subsection{Flow modeling}

The flow modeling was carried out using the Large-Eddy Simulation (LES) method for incompressible and isothermal flow implemented in Front Flow Blue 5 (Kato and Ikegawa 1991, Guo, Kato and Yamade 2006). This software allows solving, over the discretized flow domains described in Section 2.1 and Section 2.2, the filtered momentum 
equation (equation (3)) and continuity equation (equation (4)):

$$
\begin{aligned}
\frac{\partial \bar{u}_{i}}{\partial t}+\frac{\partial}{\partial x_{j}}\left(\bar{u}_{i} \bar{u}_{j}\right)= & -\frac{1}{\rho_{0}} \frac{\partial \bar{p}}{\partial x_{i}} \\
& +\nu \frac{\partial}{\partial x_{j}}\left(\frac{\partial \bar{u}_{i}}{\partial x_{j}}+\frac{\partial \bar{u}_{j}}{\partial x_{i}}\right) \\
& -\frac{\partial \tau_{i j}}{\partial x_{j}} \\
\frac{\partial \bar{u}_{i}}{\partial x_{i}} & =0, i=1,2,3,
\end{aligned}
$$

in which $\bar{u}_{i}$ and $\bar{p}$ are respectively the filtered velocity and the filtered pressure and $\tau_{i j}$ is the subgrid-scale (SGS) stress tensor (Sagaut 2005). Second-order Crank-Nicolson scheme (implicit method) is used for the discretization of the momentum equation. The unknowns $\bar{u}_{i}$ and $\bar{p}$ result from the decomposition of the velocity $u_{i}=\bar{u}_{i}+u_{i}^{\prime}$ and the pressure $p=\bar{p}+p^{\prime}$ into a resolvable part $\left(\bar{u}_{i}\right.$ and $\bar{p}$ ) and a subgrid-scale part ( $u_{i}^{\prime}$ and $p^{\prime}$ ). The stress tensor $\tau_{i j}$ groups all the terms which are not exclusively dependent on the large scale, so that:

$$
\tau_{i j}=\bar{u}_{i} \bar{u}_{j}-\overline{u_{i} u_{j}} .
$$

The method used to model the subgrid-scale turbulence is the dynamic Smagorinsky model which is based on the formulation of the subgrid-scale stress as:

$$
\tau_{i j}-\frac{1}{3} \tau_{k k} \delta_{i j}=-2 \nu_{S G S} \bar{S}_{i j}
$$

in which $\bar{S}_{i j}$ is the strain rate tensor defined by:

$$
\bar{S}_{i j}=\frac{1}{2}\left(\frac{\partial \bar{u}_{i}}{\partial x_{j}}+\frac{\partial \bar{u}_{j}}{\partial x_{i}}\right)
$$

and $\nu_{S G S}$ is the subgrid-scale eddy-viscosity defined by:

$$
\nu_{S G S}=\left(C_{S} \Delta\right)^{2} \sqrt{2 \bar{S}_{i j} \bar{S}_{i j}}
$$

in which $C_{s}$ is the Smagorinsky constant and $\Delta$ is the filter width. In the used method, the filter width is derived automatically from the mesh size, so that $\Delta$ is the cubic root of the element volume, and the Smagorinsky constant $C_{s}$ is calculated using Lilly least square procedure (Germano, Piomelli, Moin and Cabot 1991, Lilly 1992). The pressure field is computed from the velocity field using the fractional-step method.

To ensure the numerical stability of the model, the value of the time step used to carry out the numerical simulation is chosen according to the mesh size. The values corresponding to the different constriction apertures considered, and indicated in table 5, satisfy the Courant-Friedrichs-Lewy (CFL) condition. Moreover, the inlet velocity is gradually increased during 10,000 time steps. Then the flow field is simulated with 
Table 4. Physical parameters of the model.

\begin{tabular}{lll}
\hline Parameter & Symbol & Value \\
\hline Air Density & $\rho_{0}$ & $1.2 \mathrm{~kg} \cdot \mathrm{m}^{-3}$ \\
Air Kinematic Viscosity & $\nu$ & $1.5 \times 10^{-5} \mathrm{~m}^{2} \cdot \mathrm{s}^{-1}$ \\
Speed of Sound & $c_{0}$ & $340 \mathrm{~m} \cdot \mathrm{s}^{-1}$ \\
Inlet Velocity & $U_{\text {inlet }}$ & $2.4 \mathrm{~m} \cdot \mathrm{s}^{-1}$ \\
Reynolds Number & Re & 4000 \\
\hline
\end{tabular}

Table 5. Time parameters for the numerical simulation.

\begin{tabular}{lll}
\hline Constriction Height Ratio & Time Step & Simulation Time \\
$H_{C} / H(\%)$ & $\Delta_{T}(\mathrm{~s})$ & $T_{\text {sim }}(\mathrm{ms})$ \\
\hline 6 & $5 \times 10^{-7}$ & 150 \\
8 & $1 \times 10^{-6}$ & 200 \\
10 & $2 \times 10^{-6}$ & 300 \\
20 & $5 \times 10^{-6}$ & 500 \\
30 & $1 \times 10^{-5}$ & 500 \\
\hline
\end{tabular}

the constant inlet velocity for a period of time depending on the constriction aperture. The simulation time lengths $T_{\text {sim }}$ corresponding to the cases considered are indicated in table 5. The flow simulations were performed using NEC SX9 supercomputer, allowing the computation of 10,000 time steps of the models in approximately 13 hours.

\subsection{Flow-induced noise sources}

This study was focused on the analysis of the influence of the constriction aperture on the properties of the flow-induced noise generated and particularly the acoustic sources around the teeth. The presence of the teeth as an obstacle in the flow is assumed to be a strong acoustic source (Shadle 1985) and only the interactions of the flow with the surrounding walls are assumed to produce sound (Krane 2005). In this study, the overall maximum velocity obtained is $53 \mathrm{~m} . \mathrm{s}^{-1}$ (cf. table 6 ), which implies that the maximum Mach number Ma is about 0.16 . Considering $\mathrm{Ma}<<1$, it can be assumed that the power of the quadrupole sound sources of Lighthill's theory (Lighthill 1952, Lighthill 1954) is weak compared to the power of the dipole sources on the solid boundaries (Curle 1955) since the ratio between the powers of the quadrupole sources and the dipole sources is proportional to $\mathrm{Ma}^{2}$ (Goldstein 1976).

Accounting for these assumptions, it can be considered that the main noise source is strongly linked to the fluctuations of the pressure forces exerted on the walls of the teethshaped obstacle. Thus, the flow-induced noise level as well as its spectral properties can be estimated from the surface pressure predicted by the flow simulation. In order to analyze the influence of the considered parameter on the properties of the flow-induced noise source, the values $\tilde{F}$ of the time derivative of the pressure force obtained for the 
different constriction apertures are compared. This quantity is defined as:

$$
\tilde{F}(t)=\sum_{k} \frac{d p_{k}(t)}{d t} A_{k},
$$

where $p_{k}(t)$ is the pressure in each element $k$ and $A_{k}$ is the area of each element $k$. In the following, the comparison in the frequency domain is based on normalized values of $\tilde{F}$ so that:

$$
\tilde{F}_{d B}(f)=20 \cdot \log _{10}(\tilde{F}(f))-40 \cdot \log _{10}\left(U_{C}\right)
$$

where $U_{C}$ is the bulk velocity in the constriction, estimated as:

$$
U_{C}=U_{\text {inlet }} \frac{H}{H_{C}}
$$

In this study, the transformation from the time domain to the frequency domain used was a 2048 points FFT and the sampling frequency of the pressure signals was $F_{S}=50,000 \mathrm{~Hz}$, so that the frequency analysis bandwidth was about $25 \mathrm{~Hz}$.

\section{Results and discussion}

\subsection{Flow properties}

In this section, the analysis of the flow data simulated for the five constriction apertures considered is presented. Numerical flow simulation provides data for the whole flow domain described in section 2.1. The results presented in this paper are based on the flow properties extracted from $(x, y, z=0)$ plane as shown in figure 1 . Moreover the flow properties presented are averaged values over the simulation time $T_{\text {sim }}$ which varies according to the constriction aperture, as indicated in table 5.

3.1.1. Velocity field The geometrical model considered in this study includes a rectangular channel upstream of the teeth-shaped obstacle and the velocity profile applied at the inlet is uniform. Thus, the flow upstream and within the constriction was expected to be laminar. Figure 3a presents the velocity magnitude field for the constriction aperture $H_{C}=H \times 8 \%$. It can be seen from this figure that the jet formed downstream of the constriction is relatively stable in the region near the obstacle. Downstream of the channel exit, the jet becomes unstable and turbulent so that the average velocity magnitude decreases rapidly as the distance from the obstacle increases. Moreover, the jet grows slowly in its stable part and then expands over a short distance to become much wider than the constriction aperture in its turbulent part. The characteristics of the jet are very similar for all the constriction apertures considered. However, since the value of inlet velocity $\left(U_{\text {inlet }}=2.4 \mathrm{~m} . \mathrm{s}^{-1}\right)$ is used for all the geometric cases, it can be noticed that the maximum value of the average velocity magnitude increases from $11.7 \mathrm{~m} . \mathrm{s}^{-1}$ to $53 \mathrm{~m} . \mathrm{s}^{-1}$ when the constriction aperture decreases 


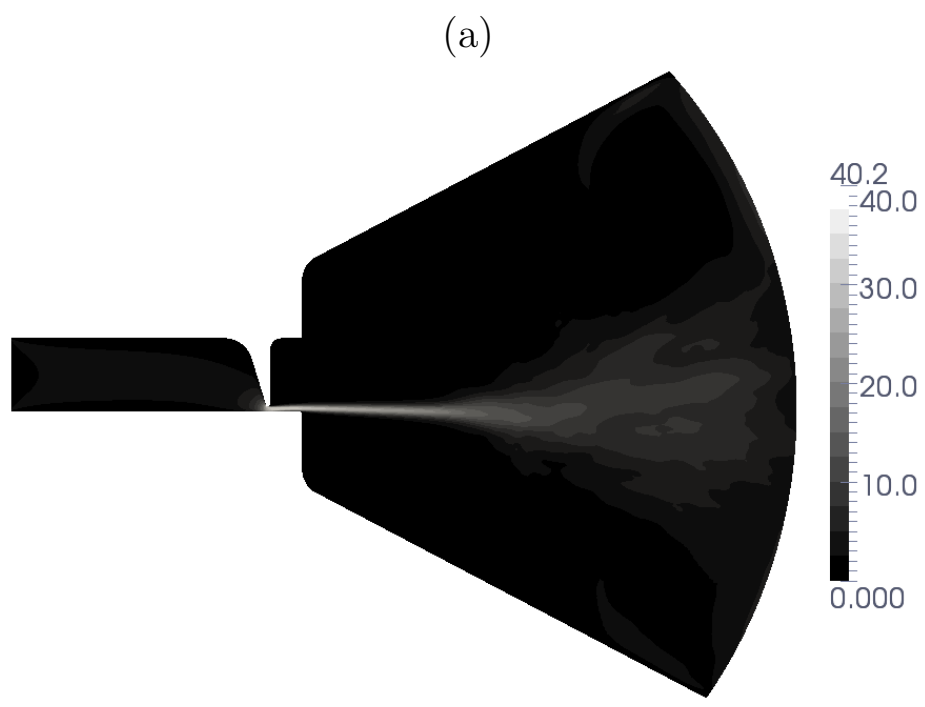

(b)

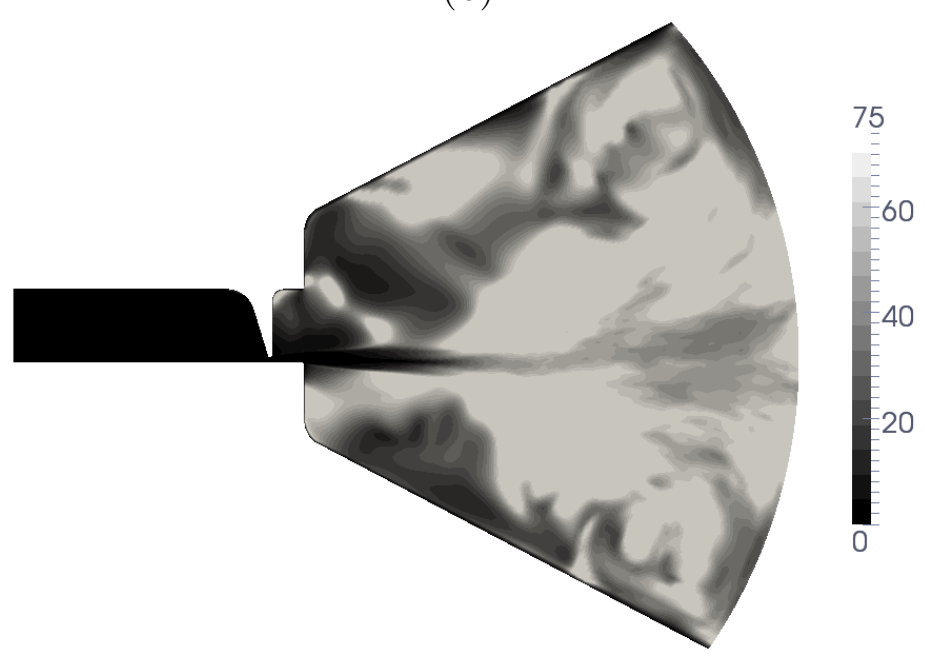

Figure 3. (a) Velocity magnitude (in $\mathrm{m} \cdot \mathrm{s}^{-1}$ ) and (b) turbulence intensity (in \%) fields in $(x, y, z=0)$ plane for the aperture $H_{C}=H \times 8 \%$.

from $H_{C}=H \times 30 \%$ to $H_{C}=H \times 6 \%$ (see table 6 ). Likewise, the length of the jet, and its stable part, decreases as the constriction aperture decreases.

The difference between the stable part and the turbulent part of the jet can be observed more clearly from the turbulence intensity field. The local turbulence intensity defined as:

$$
I_{T}=\frac{U_{r m s}}{\bar{U}}
$$

where $\bar{U}$ is the average velocity magnitude at each node and $U_{r m s}$ is the root-meansquare of the velocity fluctuations at each node, gives an indication on the strength of the turbulence field. Figure $3 \mathrm{~b}$ presents the turbulence intensity field for the constriction aperture $H_{C}=H \times 8 \%$. It can be noticed from this figure, and from figure $4 \mathrm{~b}$, that the turbulence intensity value remains below $2 \%$ in the channel upstream of the obstacle, 
Table 6. Overall maximum values of the velocity magnitude and the pressure fields.

\begin{tabular}{lll}
\hline Constriction Height Ratio & $\begin{array}{l}\text { Velocity Magnitude } \\
\left(\mathrm{m} . \mathrm{s}^{-1}\right)\end{array}$ & $\begin{array}{l}\text { Pressure } \\
(\mathrm{Pa})\end{array}$ \\
\hline 6 & 53.0 & 1316 \\
8 & 40.2 & 779 \\
10 & 32.6 & 522 \\
20 & 17.3 & 148 \\
30 & 11.7 & 72 \\
\hline
\end{tabular}

within the constriction and in the core of the jet in the region near the channel exit. This indicates that the flow can be considered as laminar in these three areas. Further downstream from the channel exit, the turbulence intensity value in the jet becomes higher than 20\%, indicating a region where the flow is highly turbulent. The regions just above and under the center of the jet where the value of the turbulence intensity is very high $\left(I_{T}>50 \%\right.$, represented in light gray and white) correspond to the jet shear layer which is caracterized by high velocity fluctuations and a low average velocity.

3.1.2. Stream-wise and span-wise velocity profiles The length of the jet downstream of the teeth-shaped obstacle decreases as the constriction becomes narrower. However, the analysis of the velocity magnitude as function of the ratio $x / H_{C}$ between the distance from the constriction and the constriction aperture shows that the evolution of the velocity magnitude is very similar for all the geometric configurations considered. Figure $4 \mathrm{a}$ presents indeed the stream-wise profiles of the velocity magnitude $U=$ $f\left(x / H_{C}\right)$, normalized by the maximum value of the profile, along the $\left(y=H_{C} / 2, z=0\right)$ axis. The reference position $x=0$ corresponds to the constriction entrance $x_{C B}$ (see figure 2). It can be noticed that all the profiles are almost superimposed even if small differences appear in the profiles shape after the maximum value. The differences come from the fact that the average jet is not strictly parallel to the axis of observation and its angle can slightly change when the constriction aperture varies. Nevertheless, for all the cases considered, the velocity magnitude reaches its maximum value within the constriction and then decreases almost linearly up to the distance $x \approx 35 \times H_{C}$.

Nevertheless, the analysis of the turbulence intensity as function of the ratio $x / H_{C}$ shows that the evolution of the turbulence intensity differs depending on the geometric configuration considered. Figure $4 \mathrm{~b}$ presents the stream-wise profiles of the turbulence intensity $I_{T}=f\left(x / H_{C}\right)$ along the $\left(y=H_{C} / 2, z=0\right)$ axis. It can be noticed from this figure that for all the constriction apertures, the turbulence is very weak $\left(I_{T}<2 \%\right)$ in the core of the jet up to the distance $x \approx 10 \times H_{C}$. Beyond this distance, two different evolutions of the profiles can be observed. For the three larger constriction apertures $\left(H_{C}=H \times\{10 \%, 20 \%, 30 \%\}\right)$, the turbulence intensity starts to increase just after the position $x \approx 10 \times H_{C}$ to reach the value $I_{T}=10 \%$ at the position $x \approx 15 \times H_{C}$. For the two smaller constriction apertures $\left(H_{C}=H \times\{6 \%, 8 \%\}\right)$, the increase in the turbulent 
(a)

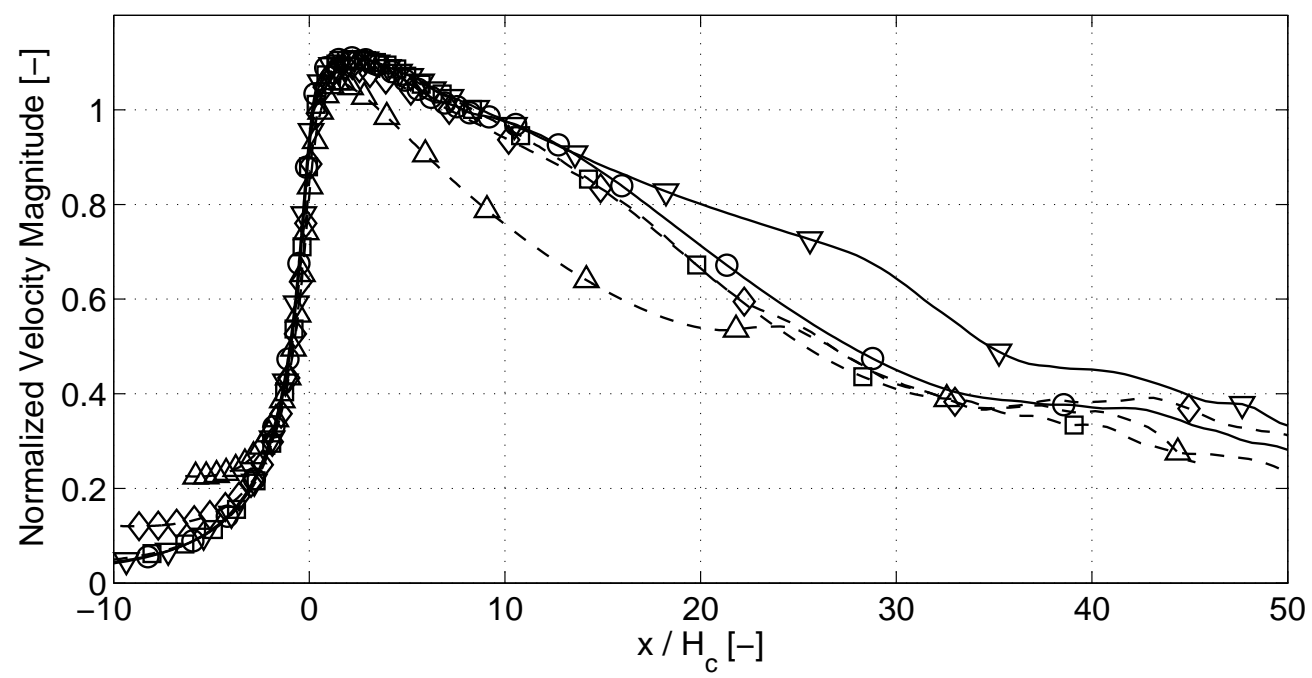

(b)

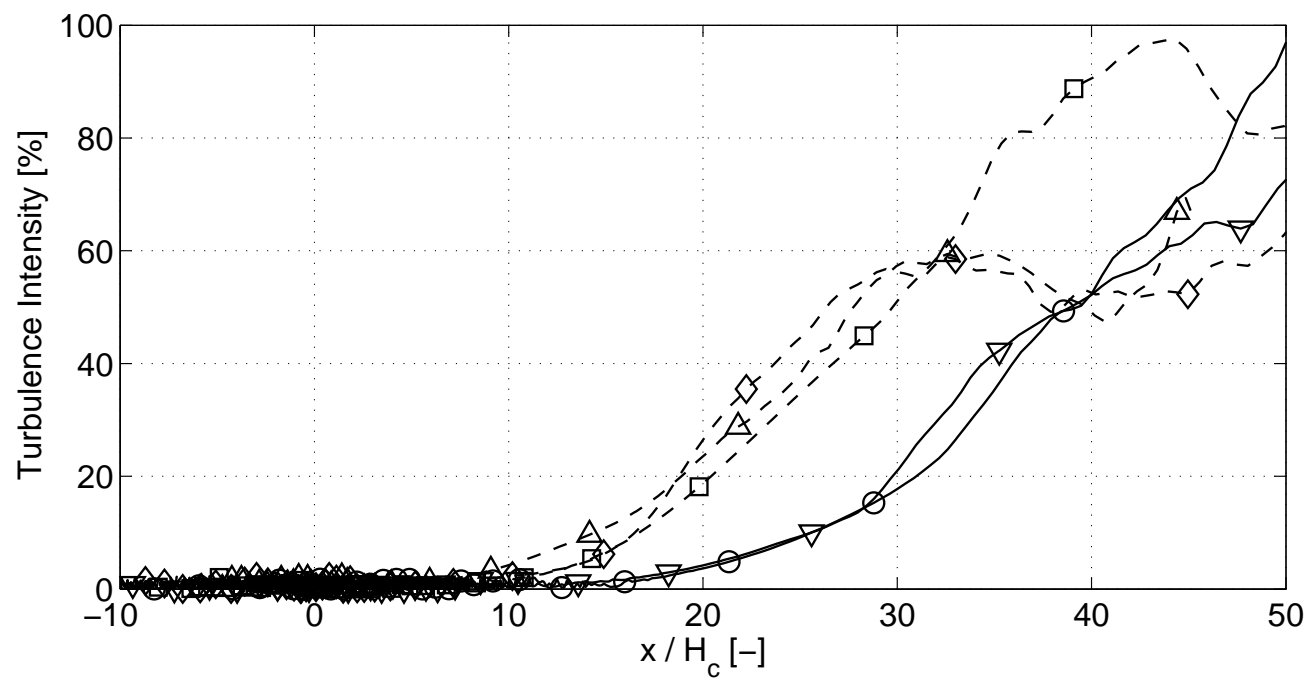

Figure 4. Stream-wise profiles of (a) the normalized velocity magnitude $\left(U=f\left(x / H_{C}, y=H_{C} / 2, z=0\right)\right)$ and (b) the turbulence intensity $\left(I_{T}=\right.$ $\left.f\left(x / H_{C}, y=H_{C} / 2, z=0\right)\right)$ for the five aperture ratios $H_{C} / H$ considered, $30 \%(\triangle)$, $20 \%(\diamond), 10 \%(\square), 8 \%(\nabla)$ and $6 \%(\bigcirc)$.

intensity starts further downstream from the obstacle and the value $I_{T}=10 \%$ is only reached at the position $x \approx 25 \times H_{C}$. Then, for all the constriction apertures, after reaching the $I_{T}=10 \%$, the turbulence intensity increases linearly up to $I_{T}=50 \%$ with the same slope. Downstream of the position corresponding to $I_{T}=50 \%$, all the profiles become different from each other. However, as mentioned in the previous paragraph, these differences can be explained by the small variations of the jet angle depending on the constriction aperture.

Therefore, the numerical flow simulations allow establishing a difference in the properties of the turbulent jet formed downstream of the obstacle depending on the 
(a)

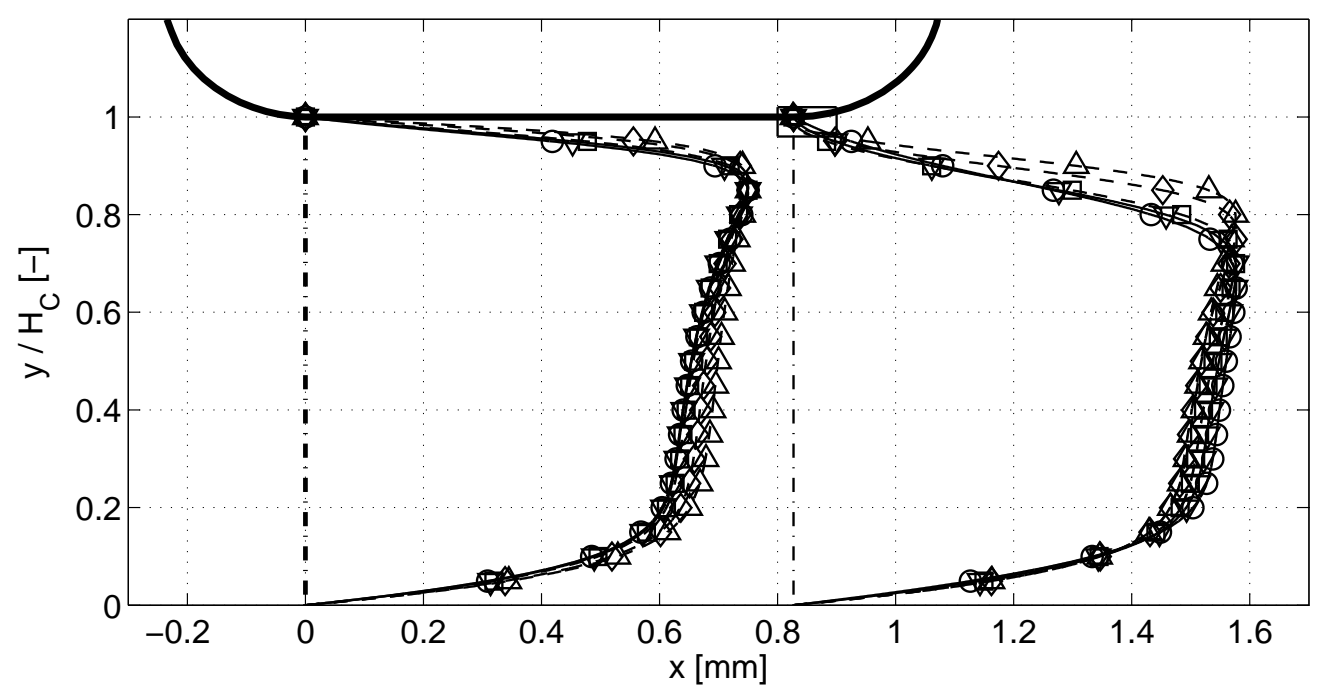

(b)

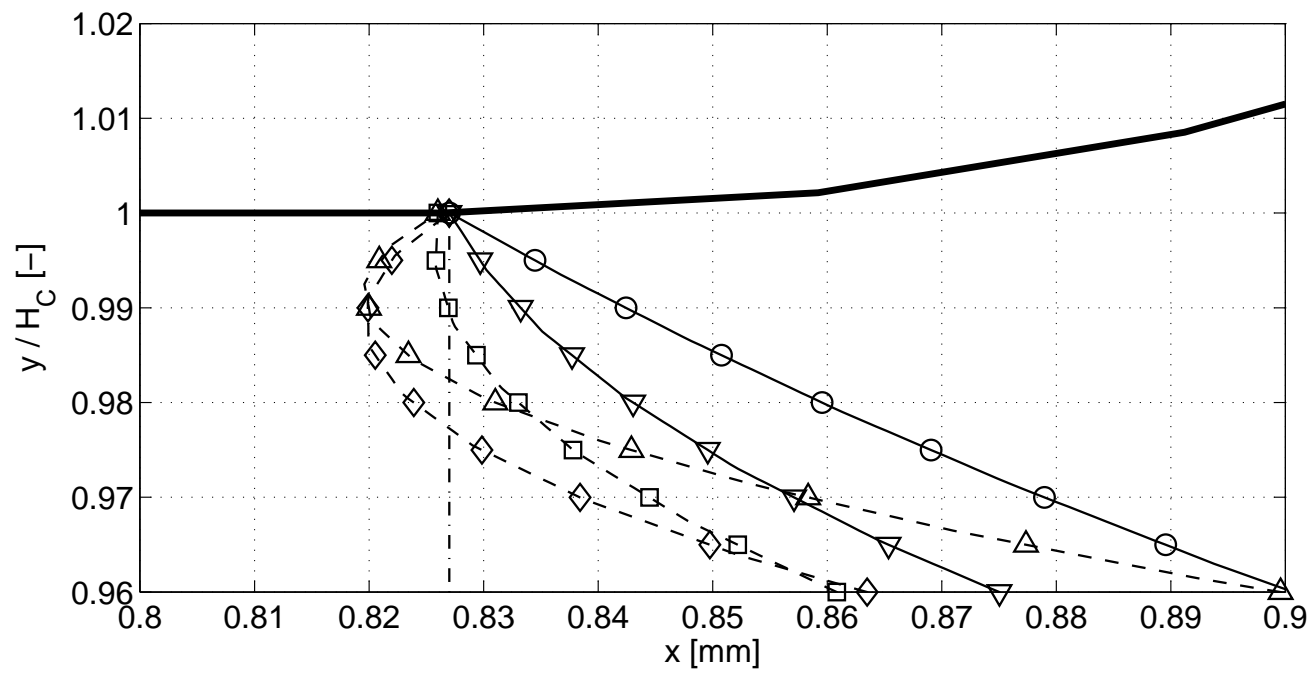

Figure 5. Span-wise profiles of the normalized average stream-wise velocity at the constriction entrance $x_{C B}$ (thick - - - ) and exit $x_{C E}$ (thin - - - ) for the five aperture ratios $H_{C} / H$ considered, $30 \%(\triangle), 20 \%(\diamond), 10 \%(\square), 8 \%(\nabla)$ and $6 \%$ (O). (b) is a zoom on the region indicated on (a). The teeth-shaped obstacle wall is indicated with thick —.

constriction aperture considered. The stable laminar part of the jet appears to be longer (considering the distance normalized by constriction aperture $x / H_{C}$ ) for the narrower constrictions corresponding to $H_{C}=H \times\{6 \%, 8 \%\}$ than for the larger apertures corresponding to $H_{C}=H \times\{10 \%, 20 \%, 30 \%\}$. This difference can result from the variations of the characteristics of the flow separation due to the teeth-shaped obstacle. Indeed, a more detailed investigation on the flow velocity within the constriction allows highlighting these variations. Figure 5 presents the span-wise profiles of the $x$-component of the velocity at the constriction entrance and exit. Thus, it can be seen that the profiles corresponding to the different constriction apertures at the entrance are 
very similar. These profiles are asymmetric and they are characterized by a maximum near the tip of the teeth-shaped obstacle. This asymmetrical shape results from the strong acceleration of the flow due to the obstacle. In return, several differences can be noticed between the profiles at the exit of the constriction. First, the shape of the profile becomes more symmetric as the constriction aperture becomes narrower. It can be seen that the velocity profile corresponding to the aperture $H_{C}=H \times 6 \%$ is almost perfectly symmetric whereas the profile corresponding to the aperture $H_{C}=H \times 30 \%$ is completely asymmetric. The shape of the profile corresponding to the aperture $H_{C}=H \times 30 \%$ at the exit of the constriction is almost the same as the shape of the profile at the entrance, except that the maximum is shifted further from the wall of the obstacle tip. The second major difference between the profiles at the exit of the constriction can be observed in figure $5 \mathrm{~b}$, which shows the part of the profiles near the wall of the obstacle tip. It can be seen that the stream-wise velocity becomes negative in the region near the wall for the two larger constriction apertures $H_{C}=H \times\{20 \%, 30 \%\}$. These negative values of the stream-wise velocity correspond to a backflow which indicates that the separation of the flow from the wall occurs upstream of the exit of the constriction. For the two narrower apertures $H_{C}=H \times\{6 \%, 8 \%\}$, no backflow is observed upstream of the exit of the constriction, indicating that the flow separation occurs downstream of the constriction. The aperture $H_{C}=H \times 10 \%$ appears to be a limit case, since the flow seems to detach from the wall upstream of the exit of the constriction as for the apertures $H_{C}=H \times\{20 \%, 30 \%\}$. However, no obvious backflow can be observed at the exit of the constriction, probably due to the accuracy of the mesh. Thus, it can be supposed that it is likely that the flow has reattached to the wall upstream of the exit of the constriction. In that case, it seems that the influence of the flow reattachment on the turbulent jet is very weak since the properties of the jet downstream of the constriction are similar to the one obtained for the apertures $H_{C}=H \times\{20 \%, 30 \%\}$.

Even if the aperture of the constriction is the only parameter varying in this study, the analysis of the velocity profiles within the constriction shows that at least two others geometric parameters defining the teeth-shaped obstacle would have an influence on the flow properties within the constriction and on the turbulent jet features downstream of the obstacle. Both the angle of the upstream edge of the obstacle and the length of the constriction would modify the flow features and in particular the flow detachment from the wall. Indeed, the angle of the upstream edge of the obstacle would modify the acceleration of the flow at the tip of the obstacle and the velocity profile at the entrance of the constriction $\left(x=x_{C B}\right)$. The length of the constriction would have an influence on the downstream turbulent jet features by changing the limit corresponding to the differences between the flow properties obtained for $H_{C}<H \times 10 \%$ and $H_{C}>H \times 10 \%$. This shows that the flow features depend on several correlated geometric parameters and illustrates the importance of a systematic analysis based on simplified geometries.

3.1.3. Pressure field The distributions of the average pressure over the flow domain corresponding to the different constriction apertures appear to be very similar. Only 


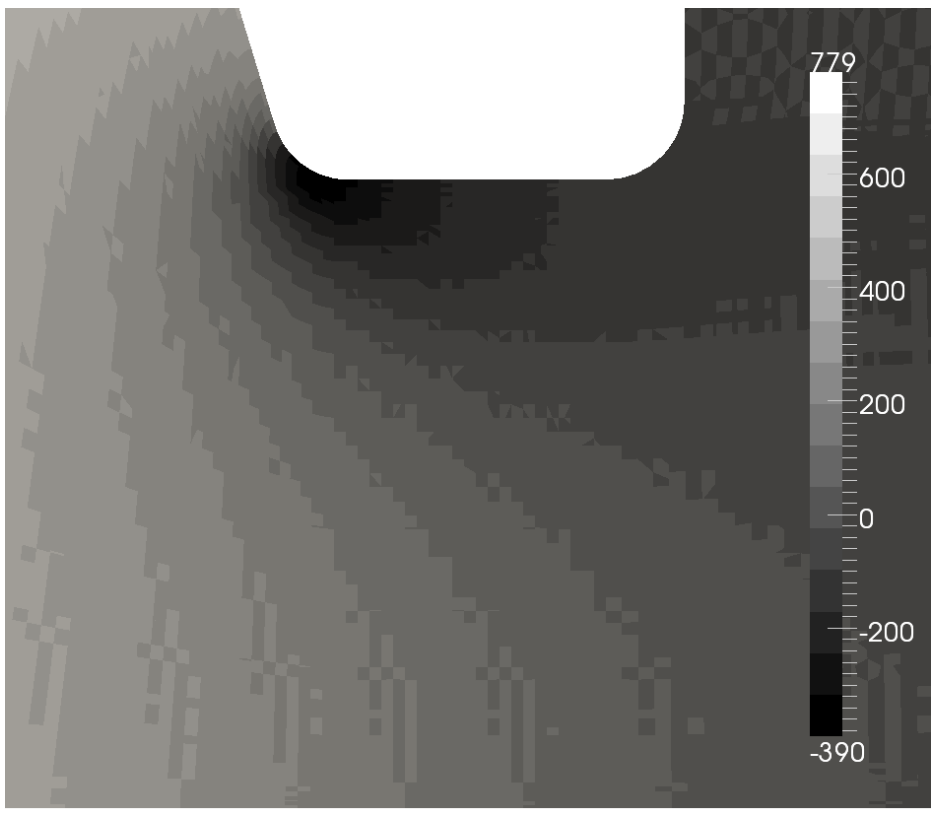

Figure 6. Pressure field (in Pa) within the constriction in $(x, y, z=0)$ plane for the aperture $H_{C}=H \times 8 \%$.

the difference between the maximum and minimum values of the pressure field increases as the constriction aperture decreases, since the imposed inlet velocity is the same for all the cases considered. The average pressure field maximum values corresponding to the different constriction apertures are reported in table 6. In the domain downstream of the constriction where the turbulent jet is located, the average pressure is relatively low compared to the upstream pressure and it oscillates around 0. The strong spatial variations of the average pressure occur in the region near the tip of the teeth-shaped obstacle. Figure 6 illustrates the average pressure field within the constriction for the aperture $H_{C}=H \times 8 \%$. It can be seen from this figure that the strong acceleration of the flow around the upstream part of the tip of the ostacle induces a high pressure drop. Thus, the negative pressure values can be observed at the entrance of the constriction near the obstacle wall. However, since the acceleration of the flow is weaker in the region near the opposite wall, the pressure remains positive. Thus, span-wise profiles of the pressure within the constriction are strongly asymmetric. Moreover, the pressure gradient is very high in the region near the wall of the teeth-shaped obstacle tip.

\subsubsection{Pressure profiles along the obstacle surface The characterization on the} pressure along the teeth-shaped obstacle surface is very important since the sound generated induced by the flow is computed from this quantity. Figure 7 a presents the profiles of the average pressure along the obstacle surface corresponding to the different constriction apertures considered. It can be seen from this figure that the profiles are similar for all the cases considered and that they admit a minimum just upstream of the entrance of the constriction. The minimum value of the average pressure $\bar{P}$ is 
(a)

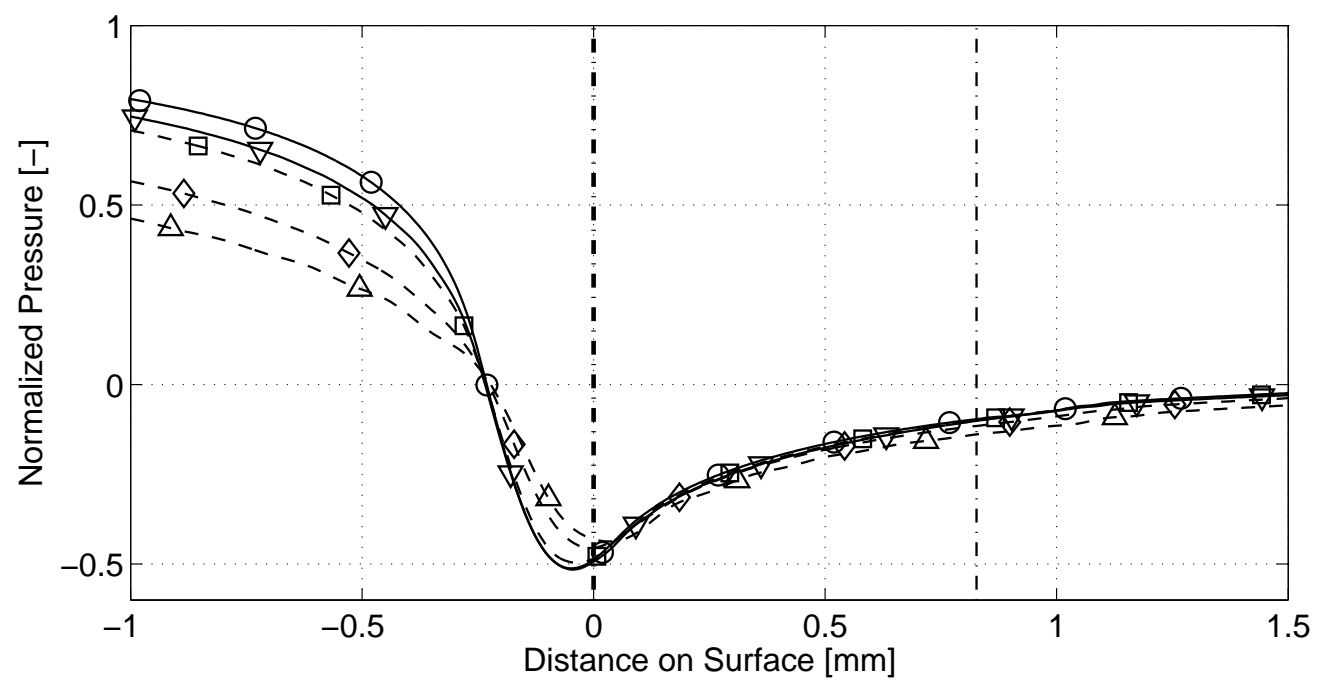

(b)

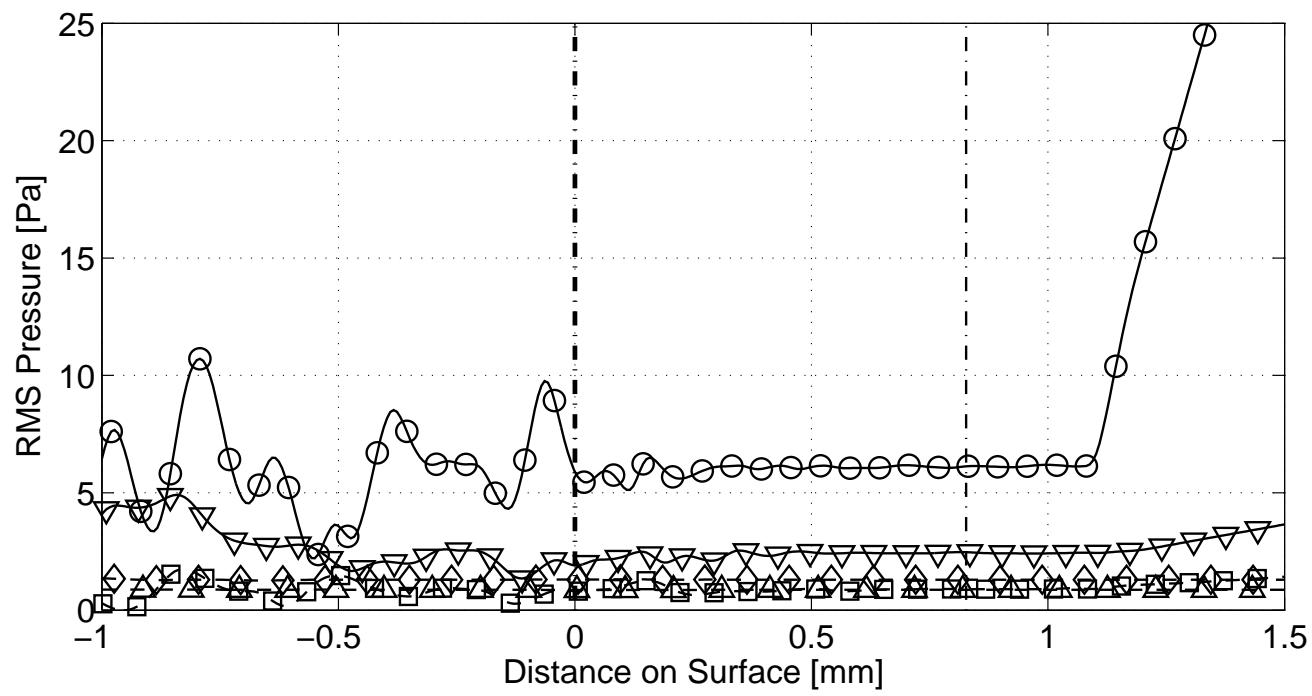

Figure 7. Profiles along the surface of the teeth-shaped obstacle (in $(x, y, z=0)$ plane) of (a) the normalized pressure and (b) the RMS pressure for the five aperture ratios $H_{C} / H$ considered, $30 \%(\triangle), 20 \%(\diamond), 10 \%(\square), 8 \%(\nabla)$ and $6 \%(\bigcirc)$. The constriction entrance $x_{C B}\left(\right.$ thick - - - ) and exit $x_{C E}($ thin - - - ) are also indicated.

proportional to the maximum value so that $\min (\bar{P}) \approx-0.5 \times \max (\bar{P})$. From this minimum, the pressure gradually increases to 0 within the constriction but only reaches 0 downstream of the exit of the constriction in the part corresponding to the vertical edge of the teeth-shaped obstacle.

The RMS pressure gives an indication of the magnitude of the pressure variations in time on the obstacle surface, which are directly involved in the sound generation. Figure $7 \mathrm{~b}$ presents the profiles of the RMS pressure along the obstacle surface corresponding to the different constriction apertures considered. Thus, it can be observed from this figure that, as the average pressure, the root-mean-square pressure also globally 
increases when the constriction becomes narrower. Moreover, for all the constriction apertures considered, three regions can be distinguished. The first region includes the upstream part of the obstacle, corresponding to the oblique edge, and the first half of the constriction. In this region, the RMS pressure fluctuates and the profiles contain very high peaks relative to their mean value. This is also the region where the pressure gradient is high (see figure 7a), so that this region is more likely to be one where the contribution to the resulting flow-induced noise would be high. The second region includes the second half of the constriction and the part of the obstacle tip immediately downstream of the constriction exit. In this region, the RMS pressure is stable and relatively low. Thus, it can be assumed that the noise generated in this region is very weak. The third region corresponds to the vertical edge of the obstacle which is oriented to the free field. In this region the RMS pressure increases and the profiles contain also some peaks as those observed in the first region. The amplitude of these peaks can be particularly high for the constriction aperture $H_{C}=H \times 6 \%$. The pressure gradient is very low in this region. However, the pressure variations in time due to the turbulent jet oscillations and the formation of vortices downstream of the obstacle can be directly observed on this part of the obstacle surface. Thus, this region is likely to be another one where the contribution to the resulting flow-induced noise would be high.

\subsection{Flow-induced noise source}

As mentioned in Section 2.4, the interactions of the flow with the surrounding walls are expected to be predominant in the production of sound. Since the dipole sound source is associated to the pressure fluctuations on the surface of the walls, the spectral properties of the dipole sound source can be estimated from the analysis in the frequency domain of the fluctuating pressure force exerted on the wall surface of the teeth-shaped obstacle. For each constriction aperture considered, the fluctuating pressure force was estimated using equation (9). Thus, the spectra of the normalized fluctuating pressure force, defined by equation (10), corresponding to the different apertures are presented in figure 8 as function of the Strouhal number St defined as:

$$
\mathrm{St}=\frac{f H_{C}}{U_{C}} .
$$

where $U_{C}$ is the bulk velocity in the constriction as defined in equation (11).

It can be noticed that the different spectra have different shapes but they are all characterized by the presence of peaks. For Strouhal numbers St $>0.2$, each spectrum contains two or three peaks located at different frequencies depending on the constriction aperture. The frequencies corresponding to the different peaks contained in one spectrum increase as the constriction becomes narrower and hence as the flow velocity increases in the constriction formed by the teeth-shaped obstacle. In each spectrum, the first peak is more pronounced than the others and as shown in figure

8 , these stronger peaks all collapse at one particular Strouhal number $\mathrm{St} \approx 0.3$. This value of Strouhal number corresponds to the typical dimensionless frequency for vortex 


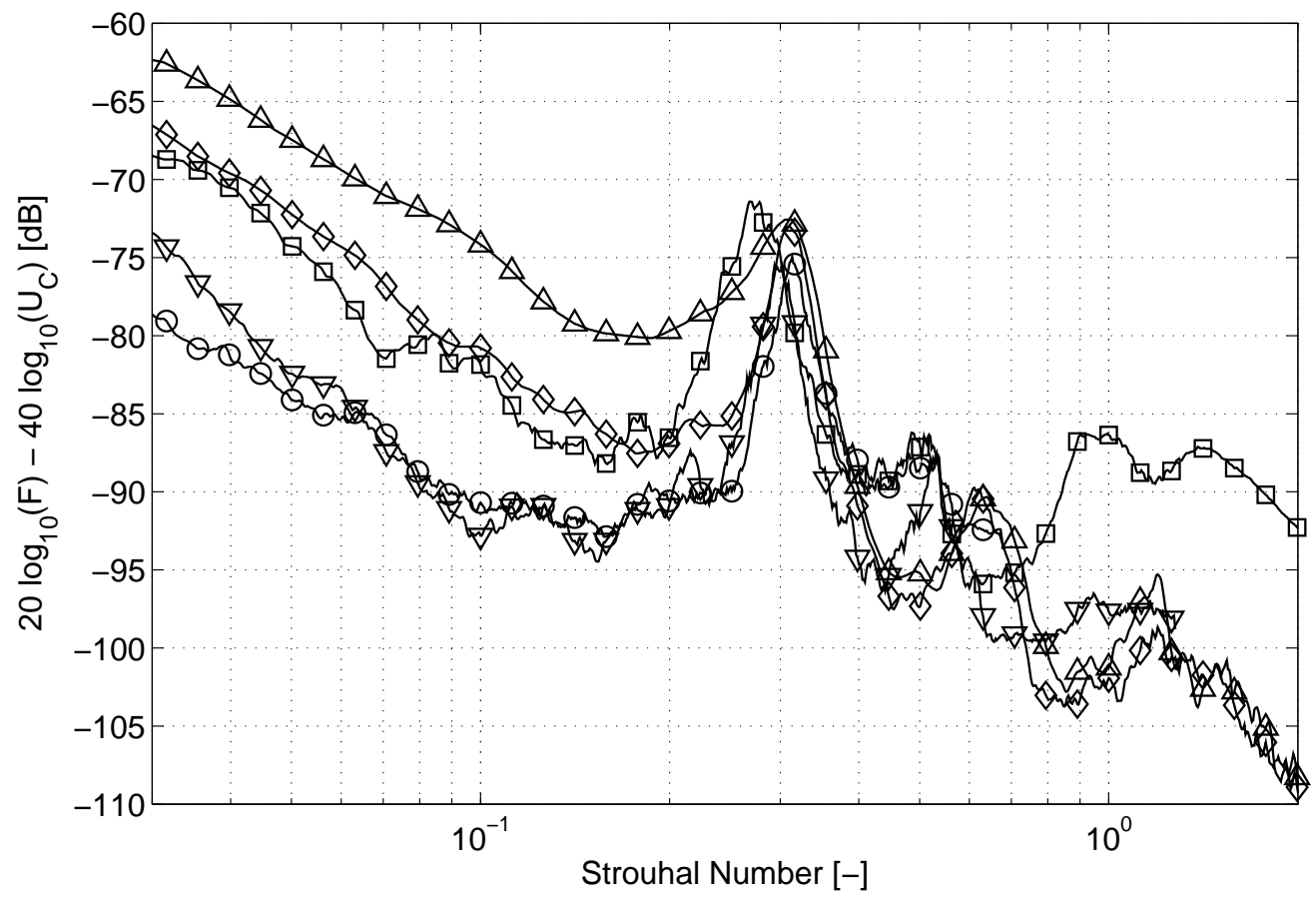

Figure 8. Spectra of the normalized fluctuating pressure force exerted on the wall surface of the teeth-shaped obstacle for the five aperture ratios $H_{C} / H$ considered, $30 \%$ $(\triangle), 20 \%(\diamond), 10 \%(\square), 8 \%(\nabla)$ and $6 \%(\bigcirc)$.

shedding from rigid objects. Such peaks were also observed by Heller and Widnall (1970) in a study of sound radiation of from flow spoilers. The frequencies of the stronger peaks coincide with the frequencies of the turbulent jet oscillations downstream of the teethshaped obstacle. The spectral analysis of the time derivatives of the velocity and the pressure around the constriction and in the far-field shows that the flow is characterized by fluctuations of which the frequency corresponds to the one observed in the fluctuating force spectra. So, the jet oscillations also produce variations in time of the flow properties within the constriction and hence of the pressure on the surface of the teeth-shaped obstacle. Thus, the modification of the constriction aperture induces some changes in the spectral characteristics of the sound source on the teeth-shaped obstacle surface. These differences result from the turbulent jet characteristics variations as function of the constriction aperture.

In addition to the flow-induced noise source spectral characteristics, the constriction aperture has a strong influence on the power of the source which globally increases when the constriction becomes narrower. The normalization of the fluctuating pressure force $\tilde{F}$ by the bulk velocity in the constriction $U_{C}$ shows that the level the stronger peak in the spectra of $\tilde{F}$ depends on $U_{C}^{4}$. Indeed, all the stronger peaks corresponding to the different constriction apertures are almost superimposed. This collapse of the main peaks in normalized frequency and normalized level is in agreement with the the $U_{C}^{4}$ dependency of the sound source level for low Strouhal number $(\mathrm{St}<1$ ) predicted by Nelson and Morfey (1981). 
Finally, for all the constriction apertures considered, the value of the fluctuating pressure force level is very low in comparison to the typical sound power level observed during the production of speech. This aspect underlines the limitations of the simplified model used in this study. Indeed, only the primary dipole sound source is considered so that this estimation of the source does not account for the amplification resulting from the acoustical resonances of the cavities, as well as the sound radiation at the exit of the duct. Moreover, as noticed in Section 3.1.1, the turbulence intensity of the flow simulated in the region upstream of the teeth-shaped obstacle and in the constriction remains very low. During the sibilant $[\mathrm{s}]$ production in real condition, the flow upstream of the teeth is already turbulent. Thus, the flow-induced sound power level is expected to be higher.

\section{Conclusions}

The research presented describes the influence of the aperture of a constriction formed by a teeth-shaped obstacle on the spectral properties of the flow-induced sound sources evaluated from the pressure fluctuations on the surface of this obstacle. It has been shown that the narrowing of the constriction leads to an increase in the resulting sound source power level. Moreover, the characteristics of the flow within the constriction and of the turbulent jet formed downstream of the obstacle change depending on the constriction aperture. Thus, the fluctuations of the turbulent jet in the free field have a direct impact on the spectral characteristics of the sound source. The simplified geometric model used in this study allows the characterization from numerical flow simulation, based on Large Eddy Simulation method, of the sound source resulting from the presence of an obstacle in a rectangular channel. This simplified geometry represents a first approach in the elaboration of a complete physical model of the sibilant [s] production, of which the reality is much more complex.

The teeth-shaped obstacle was designed from morphological incisors data. Nonetheless, the influence of only one parameter, corresponding to the aperture at the teeth, on the resulting sound source was characterized in this study. The result obtained also show that the angle of the upstream edge of the teeth-shaped obstacle and the length of the constriction formed by the obstacle can have an influence on the properties of the flow within the constriction and of the downstream turbulent jet features. Indeed, these two other geometric parameters would modify the flow detachment from the wall of the obstacle tip and consequently the stability of the jet. Thus, the influence of variations of other morphological characteristics such as the angle of the teeth, their length or their relative position, on the flow-induced sound properties should be evaluated. In the results obtained, the turbulence of the flow remains very low in comparison to measurements and observations of the real phenomenon. Moreover, the constriction in the mouth and the aperture at the teeth, which allow the production of a hissing sound like $[\mathrm{s}]$, are narrower than the apertures considered in this study. Thus, simulations with more turbulent flow, especially upstream and within the constriction formed by 
the teeth-shaped obstacle, appear to be crucial to obtain more realistic sound levels. By including the formation of a turbulent jet upstream of the obstacle in the modeling, stronger acoustic sources would appear on the surface of the obstacle surface, underlain by the interactions of the jet with the obstacle wall. Initiating the turbulence of the flow upstream of the constriction would also modify the stability and the development of the jet downstream of the obstacle. In order to capture the turbulence of the flow and to account for very small constrictions, a very fine mesh is needed to perform accurate flow simulation. Nevertheless, it is valuable to carry out numerical simulations from caricatured geometric models in order to characterize the key parameters in the mechanism of flow-induced sound generation during the sibilant $[\mathrm{s}]$ production. In addition, simplified geometries allow the experimental validation of the simulated data (Van Hirtum et al. 2010).

In this study, the spectral properties of the sound sources are only evaluated from the pressure fluctuations on the surface of the teeth-shaped obstacle. The application of computational aeroacoustic methods to analyze confined flow-induced noise generation (De Roeck 2007) would allow the characterization of the acoustical field and it would provide a more relevant modeling of the noise generated.

\section{Acknowledgments}

The authors would like to thank Masanori Nakamura (MEI Center, Osaka University, Japan) for fruitful discussions, as well as Shinji Shimojo (NICT, Japan) and Xavier Pelorson (GIPSA-lab, CNRS, France) for their support. The authors are also grateful to Cybermedia Center, Osaka University, Japan for providing access to supercomputers. This work has been supported by a postdoctoral research grant from the Japan Society for the Promotion of Science (JSPS) and by Strategic International Cooperative Program, Japan Science and Technology Agency (JST) and ANR petaflow - CSD1-ANR09-BLAN-0376-01.

\section{References}

Anderson, P., Green, S. and Fels, S.: 2009, Modeling fluid flow in the airway using cfd with a focus on fricative acoustics., 1st International Workshop on Dynamic Modeling of the Oral, Pharyngeal and Laryngeal Complex for Biomedical Applications (OPAL-2009), Vancouver, BC, Canada, pp. 146-154.

Badin, P.: 1989, Acoustics of voiceless fricatives: Production theory and data., STL-QPSR 30(3), 3355.

Catford, J. C.: 1977, Fundamental problems in phonetics., Indiana University Press, Bloomington, IN, USA.

Curle, N.: 1955, The influence of solid boundaries upon aerodynamic sound., Proc. R. Soc. Lond. 231, 505-514.

De Roeck, W.: 2007, Hybrid methodologies for the computational aeroacoustics analysis of confined subsonic flows., $\mathrm{PhD}$ thesis, Katholieke Universiteit Leuven, Belgium.

Ellis, E. and McNamara, J.: 1986, Cephalometric evaluation of incisor position., Angle Orthodontist 56, 324-344. 
Fletcher, S. G. and Newman, D. G.: 1991, [s] and [/] as a function of linguapalatal contact place and sibilant groove width., J. Acoust. Soc. Am. 89(2), 850-858.

Fredericks, C. D.: 1974, A method for determining the maxillary incisor inclination., Angle Orthodontist 44(4), 341-345.

Germano, M., Piomelli, U., Moin, P. and Cabot, W. H.: 1991, A dynamic subgridscale eddy-viscosity model., Physics of Fluids A 3(7), 1760-1765.

Goldstein, M. E.: 1976, Aeroacoustics., McGraw Hill, New York, NY, USA.

Grandchamp, X., Van Hirtum, A., Nozaki, K., Cisonni, J., Pelorson, X. and Shimojo, S.: 2010, Visualisation of moderate Reynolds number flow through a tooth-shaped nozzle., Proc. 20th International Conference on Acoustics (ICA), Sydney, Australia, p. 6 pp.

Guo, Y., Kato, C. and Yamade, Y.: 2006, Basic features of the fluid dynamics simulation software 'FrontFlow/Blue'., Monthly Journal of Institute of Industrial Science, University of Tokyo 58, 11-15.

Heller, H. H. and Widnall, S. E.: 1970, Sound radiation from rigid flow spoilers correlated with fluctuating forces., J. Acoust. Soc. Am. 47 (3), 924-936.

Howe, M. and McGowan, R.: 2005, Aeroacoustics of [s]., Proc. R. Soc. A 461, 1005-1028.

Kato, C. and Ikegawa, M.: 1991, Large Eddy Simulation of unsteady turbulent wake of a circular cylinder using the finite element method., Proc. ASME and JSME Joint Fluids Engineering Conference, Portland, OR, USA, pp. 49-56.

Krane, M. H.: 2005, Aeroacoustic production of low-frequency unvoiced speech sounds., J. Acoust. Soc. Am. 118 (1), 410-427.

Lighthill, M. J.: 1952, On sound generated aerodynamically. I. General theory. Proc. R. Soc. Lond. A 211, 564-587.

Lighthill, M. J.: 1954, On sound generated aerodynamically. II. Turbulence as a source of sound. Proc. R. Soc. Lond. A 222, 1-32.

Lilly, D. K.: 1992, A proposed modification of the Germano subgrid-scale closure model., Physics of Fluids A 4(3), 633-635.

Magne, P., Gallucci, G. O. and Belser, U. C.: 2003, Anatomic crown width/length ratios of unworn and worn maxillary teeth in white subjects., The Journal of Prosthetic Dentistry 89(5), 453-461.

Nelson, P. A. and Morfey, C. L.: 1981, Aerodynamic sound production in low speed flow ducts., J. of Sound and Vibration 79 (2), 263-289.

Nissen, S. L. and Fox, R. A.: 2005, Acoustic and spectral characteristics of young childrens fricative productions: A developmental perspective., J. Acoust. Soc. Am. 118(4), 2570-2578.

Nozaki, K.: 2005, Numerical simulations of the sibilant [s]., Conference on Turbulences, ZAS, Berlin, Germany, p. 6 pp.

Nozaki, K., Akiyama, T., Shimojo, S., Maeda, S. and Tamagawa, H.: 2005, Integration of computational fluid dynamics and computational aero acoustics on grid for dental applications., Proc. IEEE $C B M S$, p. 6 pp.

Ramsay, G. and Shadle, C.: 2006, The influence of geometry on the initiation of turbulence in the vocal tract during the production of fricatives., 7th International Seminar on Speech Production (ISSP), pp. 581-588.

Rudolph, D. J., Dominguez, P. D., Ahn, K. and Thinh, T.: 1998, The use of tooth thickness in predicting intermaxillary tooth-size discrepancies., Angle Orthodontist 68(2), 133-140.

Runte, C., Lawerino, M., Dirksen, D., Bollmann, F., Lamprecht-Dinnesen, A. and Seifert, E.: 2001, The influence of maxillary central incisor position in complete dentures on /s/ sound production., The Journal of Prosthetic Dentistry 85 (5), 485-495.

Sagaut, P.: 2005, Large Eddy Simulation for incompressible flows: an introduction., Springer, Berlin, Germany.

Shadle, C. H.: 1985, The acoustics of fricative consonants., PhD thesis, Massachusetts Institute of Technology, Cambridge, MA, USA.

Shadle, C. H.: 1991, The effect of geometry on source mechanisms of fricative consonants., J. Phon. 
19, 409-424.

Shadle, C. H., Berezina, M., Proctor, M. and Iskarous, K.: 2008, Mechanical models of fricatives based on MRI-derived vocal tract shapes., 8th International Seminar on Speech Production (ISSP), pp. $417-420$.

Sorokin, V. R.: 1994, Inverse problem for fricatives., Speech Communication 14(3), 249-262.

Van Hirtum, A., Grandchamp, X., Pelorson, X., Nozaki, K. and Shimojo, S.: 2010, Large Eddy Simulation and 'in-vitro' experimental validation of flow around a teeth-shaped obstacle., Int. J. of Applied Mechanics 2(2), 265-279. 